\title{
Three-Dimensional Structure and Dynamics of African Easterly Waves. Part III: Genesis
}

\author{
CHRIS D. THORNCROFT \\ Department of Earth and Atmospheric Sciences, University at Albany, State University of New York, Albany, New York \\ NichOLAS M. J. HALL \\ Université Paul Sabatier, LEGOS/OMP, Toulouse, France
}

GeORGE N. KILADIS

Earth System Research Laboratory, NOAA, Boulder, Colorado

(Manuscript received 25 July 2007, in final form 25 January 2008)

\begin{abstract}
This paper promotes the view that African easterly waves (AEWs) are triggered by localized forcing, most likely associated with latent heating upstream of the region of observed AEW growth. A primitive equation model is used to show that AEWs can be triggered by finite-amplitude transient and localized latent heating on a zonally varying basic state that is linearly stable. Heating close to the entrance region of the African easterly jet (AEJ) is shown to initiate AEWs downstream. The heating leads to an initial trough that reaches the West African coast about 5-7 days later, depending on the nature of the heating profile. After this, a structure that projects strongly onto the leading linear normal mode of the basic state becomes established, characterized by a number of westward-propagating disturbances that strongly resemble AEWs. The sensitivity of the forced AEWs to the nature and location of the heating profile is examined. AEWs are most efficiently triggered by heating profiles that establish lower tropospheric circulations close to the entrance region of the AEJ. In the present study, this was best achieved by lower tropospheric heating from shallow convection or upper-level heating and lower-level cooling from a stratiform precipitation profile. Both profiles have significant heating gradients in the vertical in the mid-to-lower troposphere. This triggering paradigm for the genesis of AEWs has consequences for the variability and predictability of AEWs at weather and climate time scales. In addition to the nature of the AEJ, often emphasized, it is crucial to consider the nature and variability of upstream heating triggers.
\end{abstract}

\section{Introduction}

Given the considerable body of work that has accumulated on African easterly waves (AEWs) in recent decades, it is perhaps surprising that we still lack a convincing theory for their origin. Motivated by available observations of the African easterly jet (AEJ) (e.g., Burpee 1972; Reed et al. 1977) and application of the Charney-Stern necessary conditions for instability (Charney and Stern 1962), a consensus developed in the scientific community in the 1970s that observed AEWs arise because of a mixed barotropic-baroclinic

Corresponding author address: C. D. Thorncroft, Dept. of Earth and Atmospheric Sciences, University at Albany, SUNY, Albany, NY 12222.

E-mail: chris@atmos.albany.edu instability mechanism. This consensus was strengthened by several idealized modeling studies showing that realistic-looking AEWs could develop from small amplitudes on unstable AEJs (e.g., Rennick 1976; Simmons 1977; Thorncroft and Hoskins 1994a,b; Thorncroft 1995; Paradis et al. 1995). This paper questions the paradigm promoted in these studies, namely, that AEWs arise through some "natural selection" process similar to that envisaged by Eady (1949) for midlatitude synoptic disturbances (i.e., that from a random set of atmospheric perturbations of different scales the fastest-growing normal mode will develop and eventually dominate). We argue that AEWs are in fact triggered by some localized forcing, most likely in association with latent heating upstream of the region of observed AEW growth. 
(a) zonal wind at sigma $=0.65$

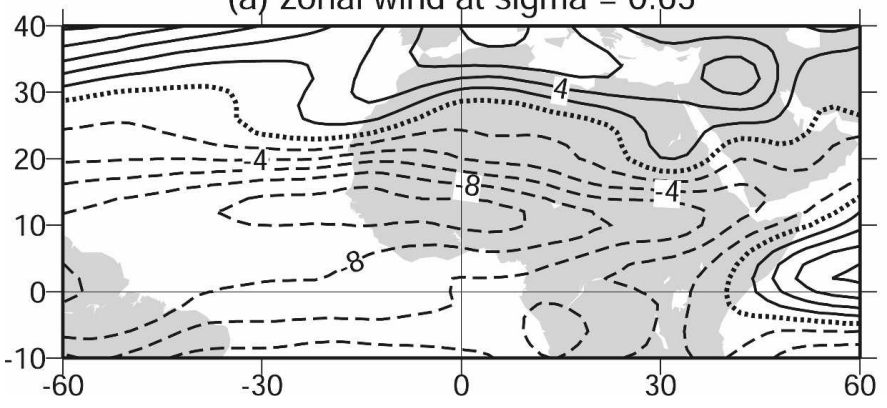

(b) vertical section at Greenwich

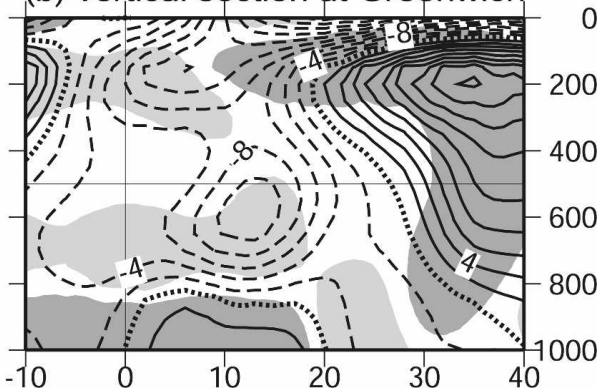

FIG. 1. Summertime (JJAS) zonal wind over West Africa from NCEP reanalyses of 1968-98: (a) at $\sigma=0.65$ and (b) the vertical meridional section at Greenwich $\left(\mathrm{lon}=0^{\circ}\right.$ ). Contours of zonal wind are every $2 \mathrm{~m} \mathrm{~s}^{-1}$; the zero contour is dotted and negative contours are dashed. Shading represents meridional wind magnitudes in excess of $0.5 \mathrm{~m} \mathrm{~s}^{-1}$. Light shading indicates northerlies; heavy shading, southerlies.

The hypothesis that AEWs grow because of a combined barotropic-baroclinic linear instability mechanism has hardly been questioned in the literature. The major weakness of this hypothesis is the observation that the AEJ is too short to support sufficient growth to explain the observed AEW amplitudes over West Africa. Considering published analyses of the AEJ (e.g., Berry and Thorncroft 2005; Kiladis et al. 2006, which is Part I of this series of papers; see also Fig. 1), we note that the region where the AEJ magnitude exceeds 6 $\mathrm{m} \mathrm{s}^{-1}$ over tropical North Africa covers approximately $40^{\circ}$ of longitude. Perhaps more importantly, the potential vorticity (PV) sign reversal in the core of the AEJ typically only extends over about $50^{\circ}$ of longitude (Dickinson and Molinari 2000). Because this represents just two wavelengths (at most) of an AEW, we suggest that it is not realistic to envisage AEWs growing through a "natural selection" process. Hall et al. (2006, hereafter HKT06), which is Part II in this sequence of papers, recently investigated the normal modes that grow on a realistic zonally varying AEJ and found that although AEWs can grow on this basic state, the growth rates are very small. With realistic surface damping the system is close to neutral stability. This observation can be seen as a further application of the ideas introduced by Farrell (1987) and Stone (1978) on the stability of the midlatitude jets. Indeed, if it is reasonable to claim that with realistic damping even the midlatitude jets are not unstable (see Hall and Sardeshmukh 1998), then it is clearly reasonable to make the same claim for the AEJ, which is weaker, shorter, and situated over the continent. So if AEWs do not grow via a linear instability mechanism, how do we account for their existence, their origin, and their intermittency? We argue that there is a growing body of work that points to the role of finite-amplitude precursors rather than AEJ instability to explain the origin of AEWs.
There have been several observational studies concerned with the origin of AEWs (e.g., Burpee 1972, 1974; Carlson 1969a, b; Albignat and Reed 1980 and references therein). Although there appears to be marked variability in the geographical origin of the AEWs, these studies generally agree that they start somewhere east of about $10^{\circ} \mathrm{E}$. Regarding mechanisms, Burpee (1972, 1974) and Albignat and Reed (1980) highlight the instability of the African easterly jet, whereas Carlson (1969a) gives more emphasis to the interactions of convection with the elevated terrain east of the Cameroon highlands $\left(\sim 10^{\circ} \mathrm{E}\right)$. Although Albignat and Reed (1980) implied that these were opposing views, we argue that they are actually not necessarily contradictory. Although AEWs rely on the presence of the AEJ to support their propagation and development, and will certainly benefit from an unstable AEJ, we maintain that it is not necessary. Whether the AEJ is unstable or not, the mechanisms of energy transfer from the jet to the waves is the same: baroclinic and barotropic conversions, which is why the structures highlighted in earlier linear instability studies remain relevant and important (e.g., Thorncroft and Hoskins 1994a). However, we contend in this paper that AEWs also rely on the presence of significant upstream convective triggers, which we expect, in general, to be linked to topography (e.g., Carlson 1969a; Hodges and Thorncroft 1997; Mekonnen et al. 2006).

In a recent case study, Berry and Thorncroft (2005) suggested that the strong AEW they observed was the result of an outburst of convection, composed of several mesoscale convective systems (MCSs), located in the mountainous region of Darfur, around $25^{\circ} \mathrm{E}$. The convective outburst clearly preceded the development of the AEW downstream. Mekonnen et al. (2006) provided a climatological perspective of the location where AEW-associated convection was initiated. They identi- 
fied two main locations for the initiation of AEWs: the Darfur mountain range and the Ethiopian highlands $\left(\sim 35^{\circ} \mathrm{E}\right)$, with the former being the most important. Kiladis et al. (2006) also showed, using time-lagged regressions, that AEWs over West Africa are preferentially preceded by convection in the Darfur region (see their Fig. 3a).

Further work is required to explore the triggering hypothesis to explain the genesis of AEWs, including more analysis of available observations together with relevant modeling studies. Indeed, recent modeling work by Hsieh and Cook $(2005,2007)$ has also highlighted the importance of convection for the genesis of AEWs. Following Schubert et al. (1991), they argue that the latent heating strengthens the potential vorticity (PV) strip on the cyclonic side of the AEJ and that the associated enhanced PV sign reversal provides a more favorable environment for AEW genesis. In the absence of the latent heating and associated PV strip in their model, AEWs fail to be generated. The mechanism is different from the mechanism being promoted here. Whereas Hsieh and Cook $(2005,2007)$ emphasize the impact of convection on the basic state that supports AEWs, the genesis mechanism explored in this paper emphasizes the potential impact of differing convective heating profiles on providing initial triggers for AEWs and sensitivity to their location with respect to the AEJ entrance region.

In this paper we pursue an idealized modeling approach that is a natural extension of the modeling approach of HKT06. We will use the same model as in that study and will investigate whether prescribed heating anomalies can trigger realistic AEWs on an observed three-dimensional basic state. The paper is organized as follows: First, we briefly describe the model and details of the approach. This is followed by analysis of modeled African easterly waves triggered by heating, including the sensitivity to the heating profile and its geographical location. We conclude with some discussion and final comments on the significance of our findings.

\section{Modeling approach}

The model and model setup used in this study is the same as that in HKT06. We utilize a global spectral primitive equation model, with a horizontal resolution of T31 and 10 equally spaced sigma levels. A semiimplicit 22.5-min time step is used to integrate the full nonlinear equations for vorticity, divergence, temperature, and log (surface pressure). A basic state is maintained by adding a forcing term that represents the combined effects of diabatic heating and transients.
The perturbation experiments are constrained to be linear by imposing a very small initial forcing perturbation and subsequently rescaling the response for presentation and discussion in this paper. In addition, a $12-\mathrm{h} \nabla^{6}$ diffusion is applied to the momentum and temperature equations. Low-level damping is included in all these simulations as described in HKT06. The average damping rates used here give time scales of about 2 days for momentum and 4 days for temperature near the surface (the coefficient actually decreases linearly from the surface, $\sigma=1$ to $\sigma=0.8$ ). In the free atmosphere (above $\sigma=0.8$ ), damping time scales for momentum and temperature are around 30 and 10 days, respectively. For further details of the modeling setup, see HKT06. The main difference between the results presented here and those in HKT06 is that here we examine a transient response to a forcing perturbation and not a pure modal structure.

We will study the atmospheric response to finiteamplitude localized heating perturbations about the mean June-September (JJAS) basic state over tropical North Africa. This is the same zonally varying basic state as used by HKT06 and is obtained from National Centers for Environmental Prediction (NCEP) reanalyses (see Fig. 1). The AEJ peaks around $12^{\circ} \mathrm{N}$ and at about $600 \mathrm{hPa}$. The peak winds are about $10 \mathrm{~m} \mathrm{~s}^{-1}$, consistent with the long averaging period. As discussed in HKT06, the weak jet is likely to result in reduced AEW growth rates compared to some shorter periods within the season when the jet is stronger. The effect of such variations in the basic state will be addressed in a subsequent contribution. Note that the AEJ weakens east of about $10^{\circ} \mathrm{E}$, highlighting the limited longitudinal extent for AEW growth over tropical North Africa.

In this study we prescribe localized heating in the model via the thermodynamic equation. In the horizontal, the heating function is given by

$$
H=H_{0} \cos ^{2} \frac{\pi}{2}\left(\frac{r}{r_{0}}\right),
$$

where $H_{0}$ is the peak heating rate at the center, $r$ is distance from the center, and $r_{0}$ determines the horizontal scale of the heating. For our basic heating run, $r_{0}$ is set to $5^{\circ}$ and the heating is centered on $15^{\circ} \mathrm{N}, 20^{\circ} \mathrm{E}$, just downstream of the Darfur mountains. For values of $r$ greater than $r_{0}, H$ is set to zero. The resulting halfwidth (the diameter where $H=H_{0} / 2$ ) for an $r_{0}$ of $5^{\circ}$ is about $540 \mathrm{~km}$. This represents a wide region of heating that, in reality, would be associated with several MCSs (Berry and Thorncroft 2005). Heating runs repeated with different radii $\left(3^{\circ}\right.$ and $\left.7^{\circ}\right)$ show differences in amplitude (not shown). The ensuing linear wave response 


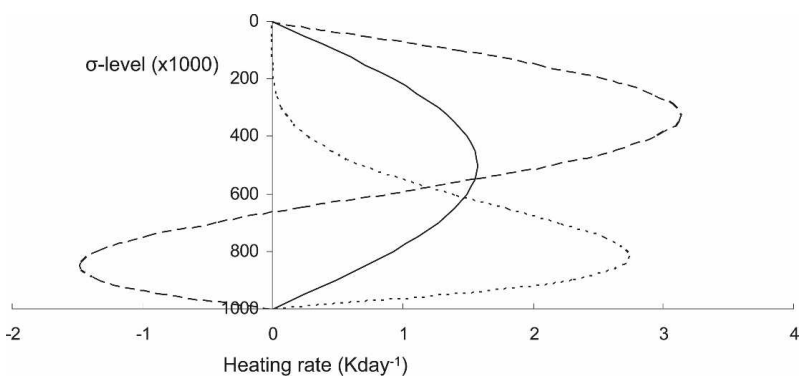

FIG. 2. Heating profiles used in this study: deep (solid), shallow (short dashed), and stratiform (long dashed). The vertical coordinate is $\sigma \times 1000$, which is approximately the same value of pressure in $\mathrm{hPa}$. Abscissas represent normalized magnitude (each profile integrates to unity in the vertical).

turns out to be proportional to the area of the initial heating perturbation, but the structure of the response is unchanged. This implies that our main results and conclusions are not sensitive to this characteristic of the heating. The vertically averaged value of $H_{0}$ used in our simulations is $5 \mathrm{~K} \mathrm{day}^{-1}$. This corresponds to a peak precipitation at the center of $20 \mathrm{~mm}$ day $^{-1}$. For the domain average with the cosine squared horizontal profile, these values are of course halved.

We will assess the sensitivity of the atmospheric response to the location and vertical profile of this heating. These profiles are approximations to those available in the literature (e.g., Schumacher and Houze (2004); see their Fig. 3a). In the vertical, the heating function that we initially consider is characterized by a deep profile to represent the effects of deep moist convection: $H_{0}=(\pi / 2) \sin (\pi \sigma)$, where $\sigma$ is the model vertical coordinate with normalized pressure (see Fig. 2). Because we know that the tropical African region is influenced by other convective types (Schumacher et al. 2004; Schumacher and Houze 2006) and that the atmospheric response to convection is sensitive to the gradient of heating along the absolute vorticity vector (e.g., Hoskins et al. 1985; Haynes and McIntyre 1987), we will explore the impacts of two other heating profiles. These include a shallow heating profile,

$$
H_{0}=\left(\frac{1}{\pi}-\frac{12}{\pi^{3}}+\frac{48}{\pi^{5}}\right)^{-1} \sigma^{4} \sin \pi \sigma,
$$

and a "stratiform" heating-cooling profile,

$$
H_{0}=\frac{75 \pi}{\left(74-18 e^{-2 \pi}\right)}\left[e^{-2 \pi(1-\sigma)}-1\right] \cos \frac{3 \pi}{2}(1-\sigma),
$$

also included in Fig. 2. The latter profile is meant to mimic the heating over cooling region dominant in regions of stratiform precipitation trailing large-scale systems of propagating deep convection (Houze 1997,
2004), whereas the former accounts for the leading edge of shallow convection within such systems (e.g., Mapes et al. 2006). All three profiles integrate to unity between $\sigma=0$ and $\sigma=1$ and so have the same total heat input to the system. The atmospheric response to these profiles is presented in section $3 b$.

A basic run consists of prescribing the heating in a particular location for a period of one day and then switching it off. The model is integrated for several days to see the adiabatic response to this. We do not include any heating within the developing AEW and so we expect the AEW amplitudes to be weaker than observed or seen in models with more complete physics (e.g., Berry and Thorncroft 2005; Hsieh and Cook 2005). The main objective here is to consider whether upstream heating can indeed lead to realistic AEW activity downstream and to investigate the impact of varying the nature of this heating, in terms of its profile and location.

\section{Forced African easterly waves}

\section{a. African easterly waves forced by localized deep heating}

The basic run that we will discuss first is forced by localized deep heating centered at $15^{\circ} \mathrm{N}, 20^{\circ} \mathrm{E}$, just downstream of the Darfur region and also close to the entrance region of the AEJ (Fig. 1a). The atmospheric response to this heating is shown here with the streamfunction at $\sigma=0.85$ (Fig. 3). We should first note that, after an initial large-scale adjustment to the heating, the atmospheric response takes the form of enhanced and coherent AEW activity in the downstream AEJ, going some way toward supporting our original hypothesis that AEWs can be forced by upstream heating.

The heating directly spins up an initial trough (Fig. 3a) that propagates westward along the AEJ, reaching around $10^{\circ} \mathrm{W}$ at day 5 of the simulation (Fig. $3 \mathrm{c}$ ). The initial atmospheric response to the heating (not shown) is baroclinic, as expected, with the establishment of a trough below the heating maximum and a ridge above. By day 1, interactions with the basic state result in a slightly more complicated ridge structure characterized by a double maximum. After day 5 , we continue to see a progression of AEWs, and associated troughs and ridges, moving westward. The structure of these perturbations is remarkably similar to those from observations of AEWs in Kiladis et al. (2006).

The first trough is rather like the trough seen in the Berry and Thorncroft (2005) observational study, where finite-amplitude heating over Darfur leads directly to a westward-propagating and growing disturbance. The observed trough in that study had a peak 

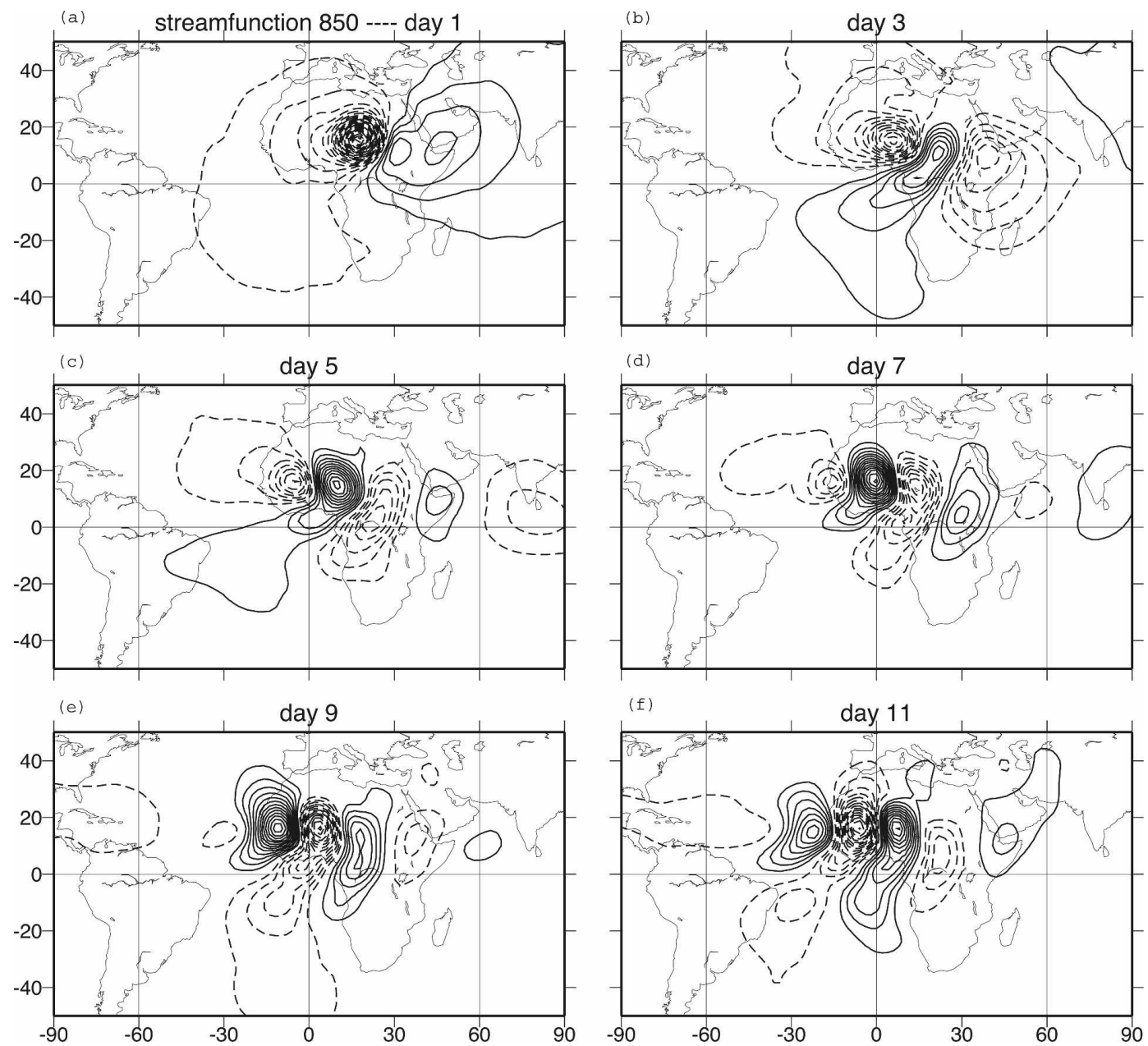

FIG. 3. Streamfunction at $\sigma=0.85$ for days $1,3,5,7,9$, and 11 for the response to a deep convective anomaly at $15^{\circ} \mathrm{N}, 20^{\circ} \mathrm{E}$. Contours are every $10^{5} \mathrm{~m}^{2} \mathrm{~s}^{-1}$; negative contours are dashed and zero is omitted.

meridional wind of about $20 \mathrm{~m} \mathrm{~s}^{-1}$ at the level of the AEJ. The maximum meridional wind perturbation at the level of the AEJ for the trough at day 5 is only about $1 \mathrm{~m} \mathrm{~s}^{-1}$ (Fig. 4b), but it should be noted that observed strong AEWs in this region are often convectively active and that this coupling with convection probably enhances their amplitudes (Hsieh and Cook 2005,2007 ), which is a process lacking in our simulations. In the absence of convection, this trough weakens as it moves out over the Atlantic, consistent with Rossby wave dispersion on a single-signed PV gradient. In the simulation, a second trough reaches $10^{\circ} \mathrm{W}$ on about day 11 (Fig. 3f), or 6 days after the first, and is in fact about twice as intense. Indeed, all subsequent waves are much stronger than the first (see Fig. 4, left column).

The forced waves that follow the initial AEW trough move westward with a phase speed of about $5^{\circ}$ day $^{-1}$, a wavelength of about $3000 \mathrm{~km}$, and horizontal and ver- tical tilts characteristic of baroclinic and barotropic growth (see day-9 fields in Figs. 3 and 4). These wave characteristics are very similar to observations and to the linear normal mode structure described in HKT06 (see their Fig. 4). Given this, we hypothesize that the prescribed heating not only caused the initial westwardmoving trough but also forced the leading linear normal mode to this zonally varying basic state.

This simulation provides strong evidence to support the hypothesis that AEWs can be forced by upstream convective heating. However, the amplitudes are rather weak, and some coupling between the AEW dynamics and convection is probably required to make up for this, as discussed by Hsieh and Cook $(2005,2007)$. Nevertheless, the fact that disturbances with quite realistic structures are produced, even without feedback from the convective heating, is certainly suggestive that AEWs can be triggered by such heating. The following subsections explore the sensitivity of the downstream 

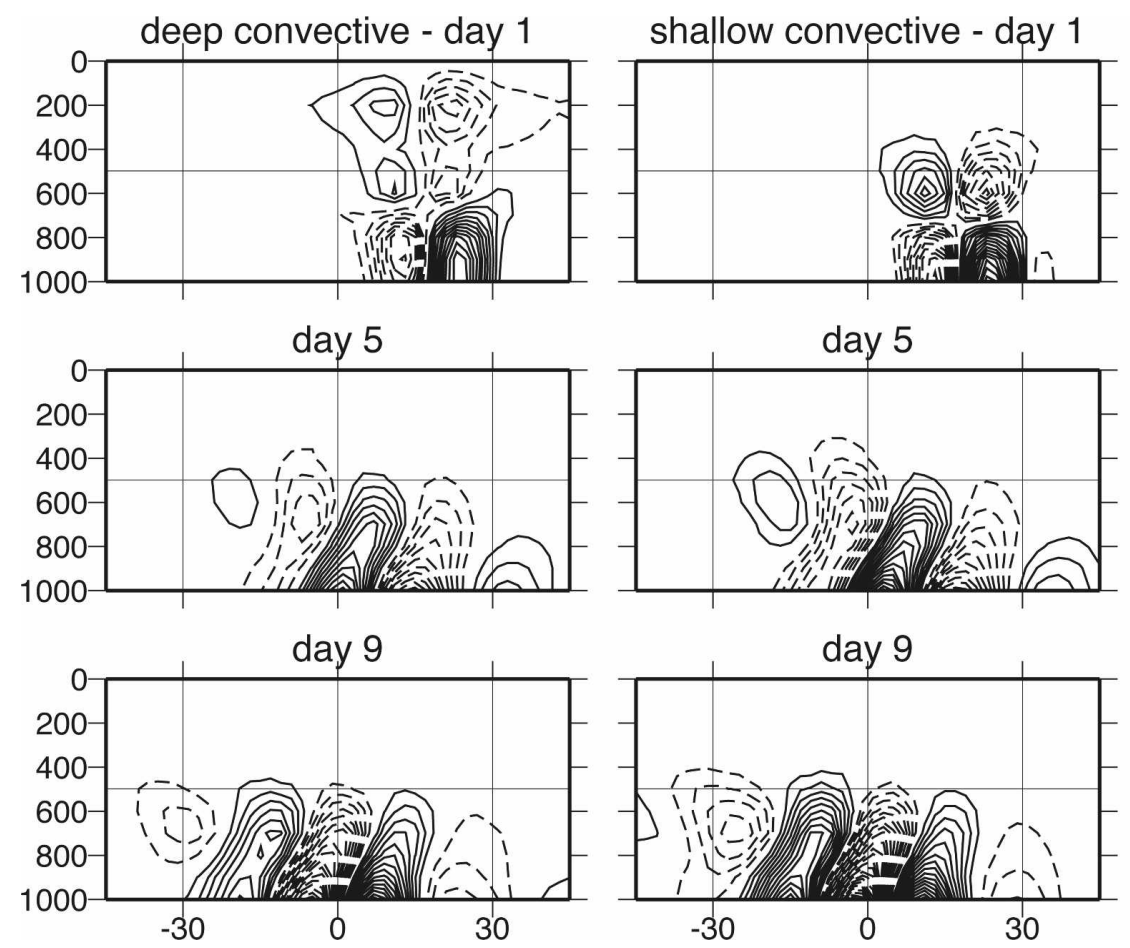
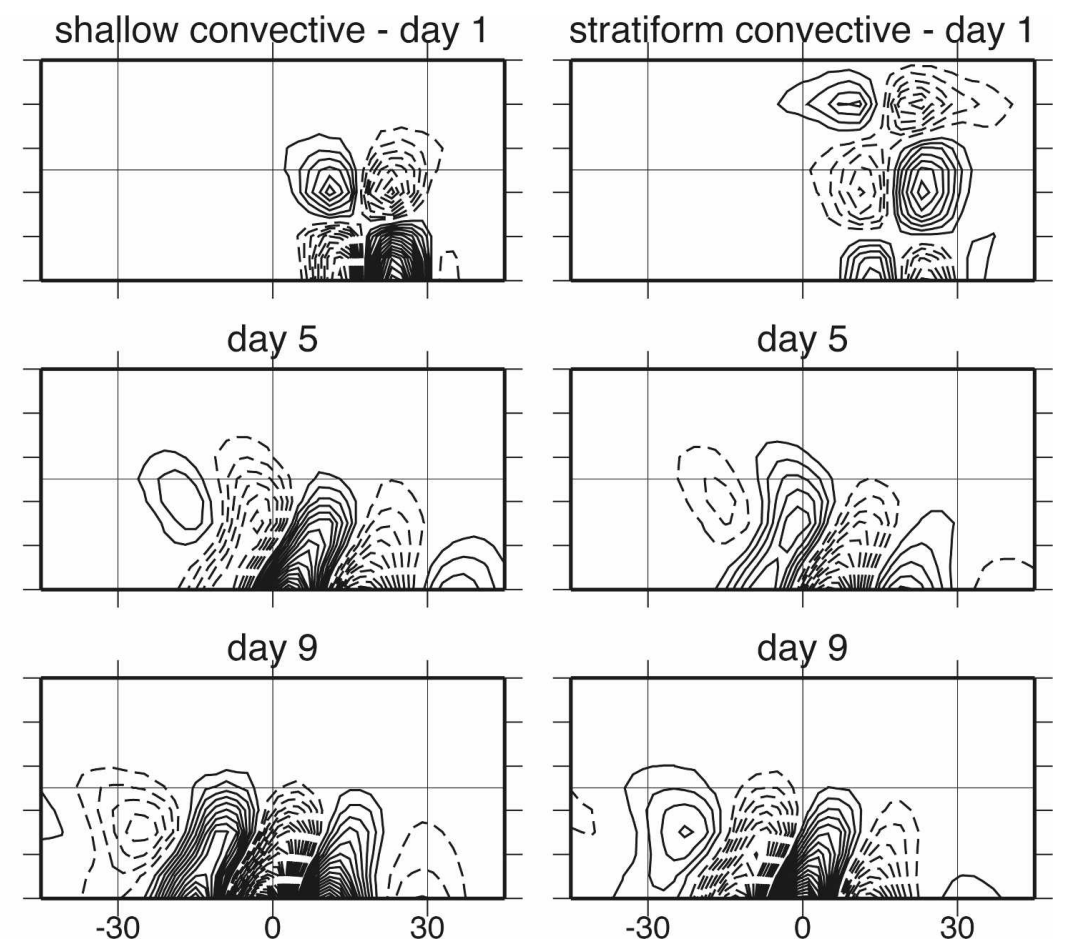

FIG. 4. West-east vertical cross section of meridional wind at $15^{\circ} \mathrm{N}$ for days 1,5 , and 9 for the three heating profiles. Contours are every $0.25 \mathrm{~m} \mathrm{~s}^{-1}$ (deep profile) and $0.5 \mathrm{~m} \mathrm{~s}^{-1}$ (shallow and stratiform profiles), with negative contours dashed and zero omitted. The vertical coordinate is $\sigma \times 1000$, which is approximately the same value of pressure in $\mathrm{hPa}$.

response to the heating profile and the location of the heating.

\section{b. Sensitivity to heating profile}

The basic simulation presented above has been repeated with two additional heating profiles (see Fig. 2) in recognition of the different populations of convecting clouds that characterize the tropical North African region (e.g., Schumacher et al. 2004; Schumacher and Houze 2006). The peak heating rates for the deep, shallow, and stratiform profiles are $1.6 \mathrm{~K} \mathrm{day}^{-1}$ at $\sigma=0.5$, $2.7 \mathrm{~K} \mathrm{day}^{-1}$ at $\sigma=0.8$, and $3.1 \mathrm{~K} \mathrm{day}^{-1}$ at $\sigma=0.35$, respectively. In addition, the stratiform profile has a peak cooling rate of $-1.5 \mathrm{~K} \mathrm{day}^{-1}$ located at $\sigma=0.85$. In reality, heating profiles generally reflect various combinations of heating structures from different types of clouds such as shallow, congestus, and deep convection, as well as stratiform precipitation (e.g., Schumacher et al. 2004; Mapes et al. 2006). This often results in "top heavy" heating profiles averaged over the scale of disturbances within the tropics, with a maximum heating in the upper troposphere and weak heating or even cooling near the surface. Because all the simulations presented here are linear, the response to a mean heating profile that includes contributions from each of the heating profiles can be constructed by summing the simulations forced by each profile with appropriate weightings (Schumacher et al. 2004). We can thus interpret, for example, the "stratiform" response as being similar to a weighted difference between the responses to deep and shallow convection.

\section{1) Shallow heating}

The evolution of the streamfunction at $\sigma=0.85$ for the simulation with shallow heating is shown in Fig. 5. As before, there is an initial adjustment to the heating that includes the triggering of a trough, reaching $10^{\circ} \mathrm{W}$ around day 7 , and thereafter the establishment of a structure that projects strongly onto the leading normal mode. The 2-day delay in reaching $10^{\circ} \mathrm{W}$, compared to the deep heating case, is due to the difference in the initial atmospheric response to the heating. In the shallow heating case, the initial triggered trough is seen clearly at day 5 near the Greenwich meridian, and this is substantially stronger than its counterpart in Fig. 3c, as are all of the other eddies. This feature also displays a much deeper vertical extent in Fig. 4e, and is a result of the combination of a low-level trough that forms below the heating and a trough at the level of the AEJ that forms upstream of the forced ridge above the heat- 

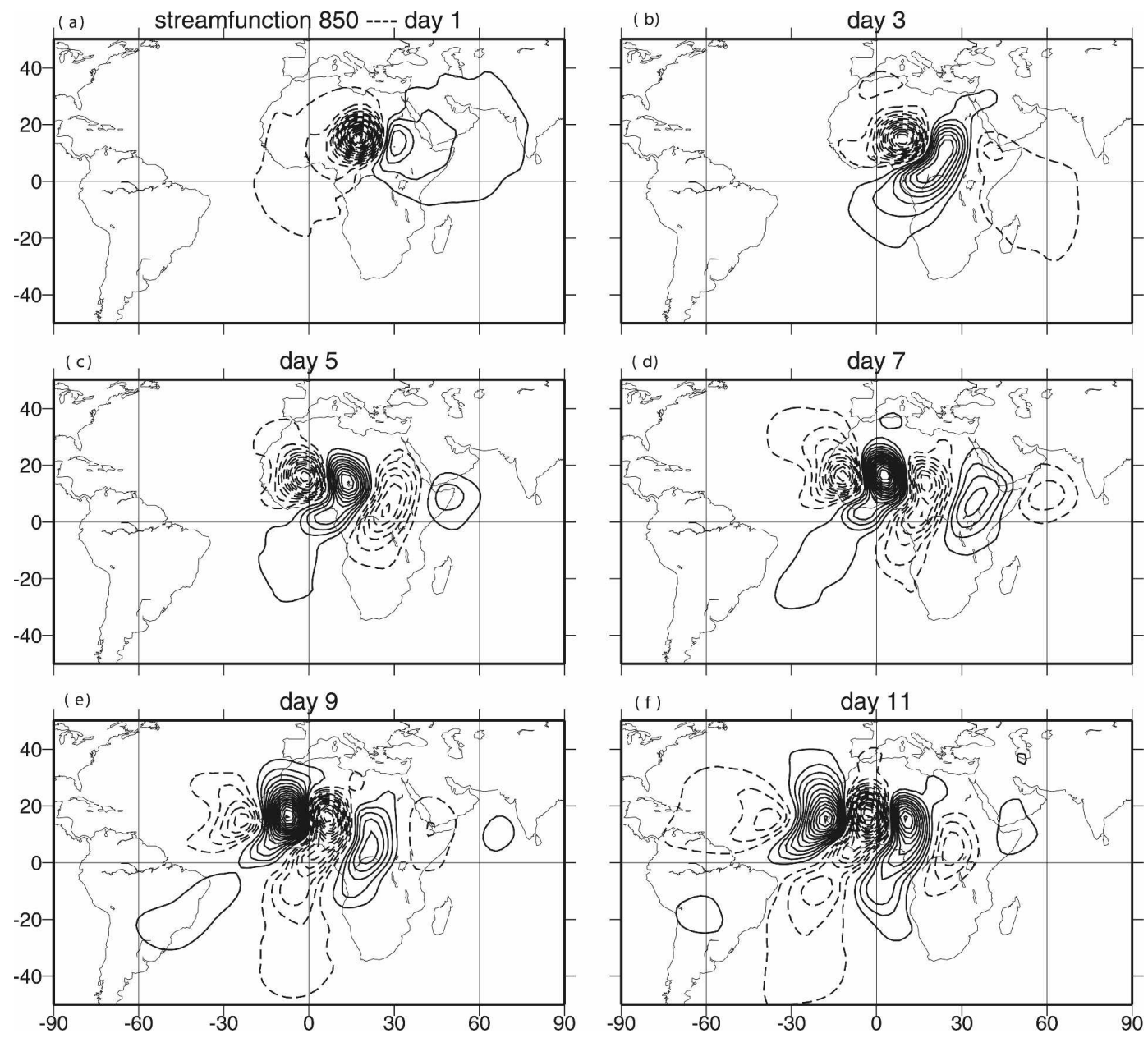

FIG. 5. As in Fig. 3, but for the response to a shallow convective anomaly. Contours are every $2 \times 10^{5} \mathrm{~m}^{2} \mathrm{~s}^{-1}$; negative contours are dashed; zero is omitted.

ing (Fig. 4d). The AEJ-level ridge and upstream trough are not present in the early days of the deep heating simulation because of the different heating profile. Instead, the upper-level ridge is located around $200 \mathrm{hPa}$ and quickly disperses with no significant upstream trough formation.

It is interesting that, despite the fact that the columnaveraged heating is the same in both cases, the initial trough and the normal mode structure that is established after day 5 are much more intense than in the simulation with deep heating. For example, the peak meridional winds at the lowest model level at days 5 and 9 in the deep heating run are 2.8 and $4.4 \mathrm{~m} \mathrm{~s}^{-1}$, respectively, compared with 7.9 and $12.1 \mathrm{~m} \mathrm{~s}^{-1}$ for the shallow heating run. The simplest explanation for this is that the shallow heating profile creates a more intense initial perturbation that overlaps more strongly with the AEJ and its associated PV gradients. Stronger lower tropospheric heating gradients would generate more in- tense PV anomalies there (Hoskins et al. 1985; Haynes and McIntyre 1987). This is confirmed in Fig. 4, which shows the meridional wind structures at $15^{\circ} \mathrm{N}$ after 1 day of heating. In the deep heating case, there is a low-level trough with peak winds of $3.2 \mathrm{~m} \mathrm{~s}^{-1}$ located around $900 \mathrm{hPa}$ and an upper-level ridge with peak winds of $1.4 \mathrm{~m} \mathrm{~s}^{-1}$ located at about $200 \mathrm{hPa}$. In the shallow heating case, the heating gradients in the vertical are much larger and confined in the lower troposphere (Fig. 2) and, consistent with this, the peak meridional winds at $15^{\circ} \mathrm{N}$ at day 1 are 8.0 and $4.2 \mathrm{~m} \mathrm{~s}^{-1}$ at 900 and $600 \mathrm{hPa}$, respectively.

We therefore conclude that for the same amount of heating the shallow heating leads to a stronger AEW response than that seen with the deep heating. An alternative way of viewing this is that shallow heating can more efficiently trigger AEWs than deep heating because of its proximity to the AEJ. However, whether shallow heating is more important than deep heating 

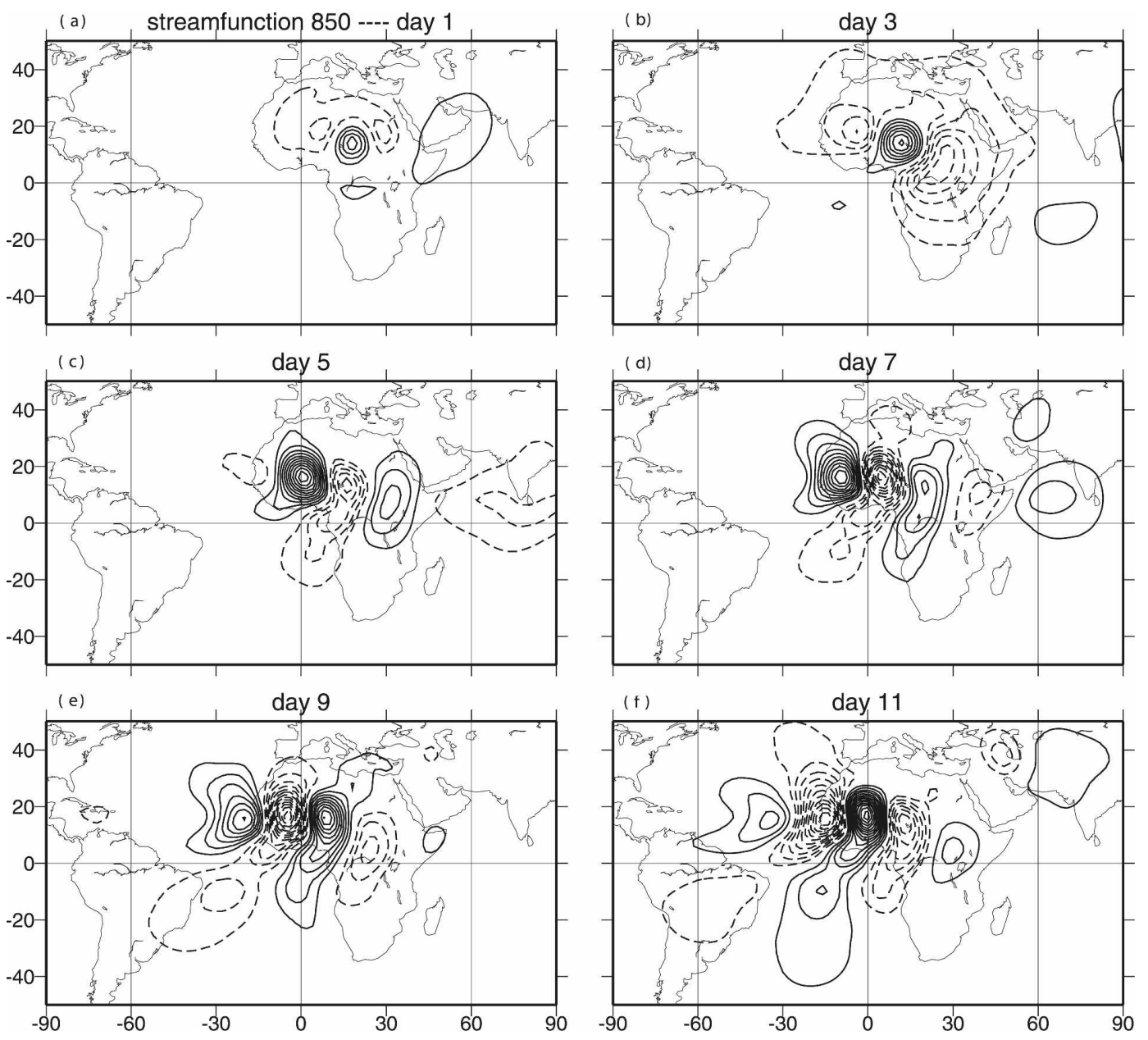

FIG. 6. As in Fig. 3, but for the response to a stratiform convective anomaly; contours as in Fig. 5.

for triggering AEWs in reality is uncertain. For example, although the deep heating is less efficient than shallow heating for triggering AEWs (for the same mean heating rate), in reality we would expect the rainfall and associated heating rates to be more intense for deep convection. Future work will explore the nature of the observed upstream convection and the resulting AEW genesis to shed more light on this issue.

\section{2) Stratiform HEATING}

In recognition of the fact that MCSs in the tropics are often dominated by stratiform precipitation, we also consider the impact of a heating profile that is characterized by heating aloft and cooling below (see Fig. 2). Hertenstein and Schubert (1991) showed that such a profile was particularly efficient at generating cyclonic PV anomalies in the midtroposphere due to the strong heating gradients in the vertical there. We therefore expect that this profile will have a strong impact on the forced AEWs and the initial trough in particular.

The initial atmospheric response to this profile is very different from the deep and shallow heating cases (Figs. 4a,d,g) and in fact is virtually opposite to the shallow profile in the mid-to-lower troposphere. As expected, a midlevel trough forms in the region where heating gradients increase with height above the cooling (Fig. 2), and a surface ridge forms beneath the cooling maxima. The evolution of the streamfunction at $\sigma=$ 0.85 for the stratiform heating case is shown in Fig. 6 . This evolution resembles the previous two in the sense that there is an initial low-level trough development followed by the normal mode, but again there are substantial differences in timing and amplitude. The initial trough reaches $10^{\circ} \mathrm{W}$ about a day earlier than the equivalent trough in the deep case, consistent with the initial trough being located closer to the AEJ peak around $600 \mathrm{hPa}$. This results in a phase difference be- 
tween the stratiform and the shallow cases of about half a period, highlighted by the day- 5 and day- 9 responses, which are almost identical except for the sign difference (cf. Figs. 4e,h and Figs. 4f,i)

The AEW amplitudes for the stratiform case are again much larger than for the deep heating case, being comparable to those for the shallow heating case (Fig. 4). As with shallow heating, the simplest explanation for this is the fact that the stratiform heating profile forces a more intense initial perturbation in the vicinity of the AEJ.

In summary, the precise shape of the heating profile is important for determining the nature of the initial trough in terms of its amplitude and downstream propagation characteristics. Also, although the subsequent forced normal mode structure appears not to be overly sensitive to the heating profile, the amplitude clearly is. A heating profile that forces more intense initial lower tropospheric circulations results in larger amplitudes.

\section{c. Sensitivity to location of heating}

The previous sections prescribed the heating in one fixed location $\left(15^{\circ} \mathrm{N}, 20^{\circ} \mathrm{E}\right)$ that was motivated by previous observational work (e.g., Berry and Thorncroft 2005; Mekonnen et al. 2006; Kiladis et al. 2006). Given that convective heating occurs over a wide region of tropical North Africa, we now investigate the impact of changing the location of the heating on the AEW response. One outcome of this will be the identification of the "most sensitive" location for forcing AEWs on this basic state. A large number of simulations were carried out with initial heating locations spanning the entire globe, but with increasingly more closely spaced intervals (up to $5^{\circ}$ of longitude and latitude) over the West African region.

To summarize these results, we show influence functions for the three heating profiles. The influence function is defined as the root-mean-square streamfunction at $\sigma=0.85$ on day 10 of the simulation over an AEW target area: $5^{\circ}-25^{\circ} \mathrm{N}, 60^{\circ} \mathrm{W}-60^{\circ} \mathrm{E}$. This value is plotted in Fig. 7 in the position of the heating perturbation that gave rise to it, leading to an identification of which areas are the most sensitive for convective generation of AEWs. The patterns are very similar for each heating profile, but the amplitudes are larger for the shallow and stratiform heating, consistent with the analysis above. It turns out that the best location to force AEWs with any of the convective heating profiles is around $20^{\circ} \mathrm{N}, 15^{\circ} \mathrm{E}$. This is close to the entrance region of the African easterly jet and just north and west of the location used in the basic simulations. The influence function falls off rapidly as the location of the heating is
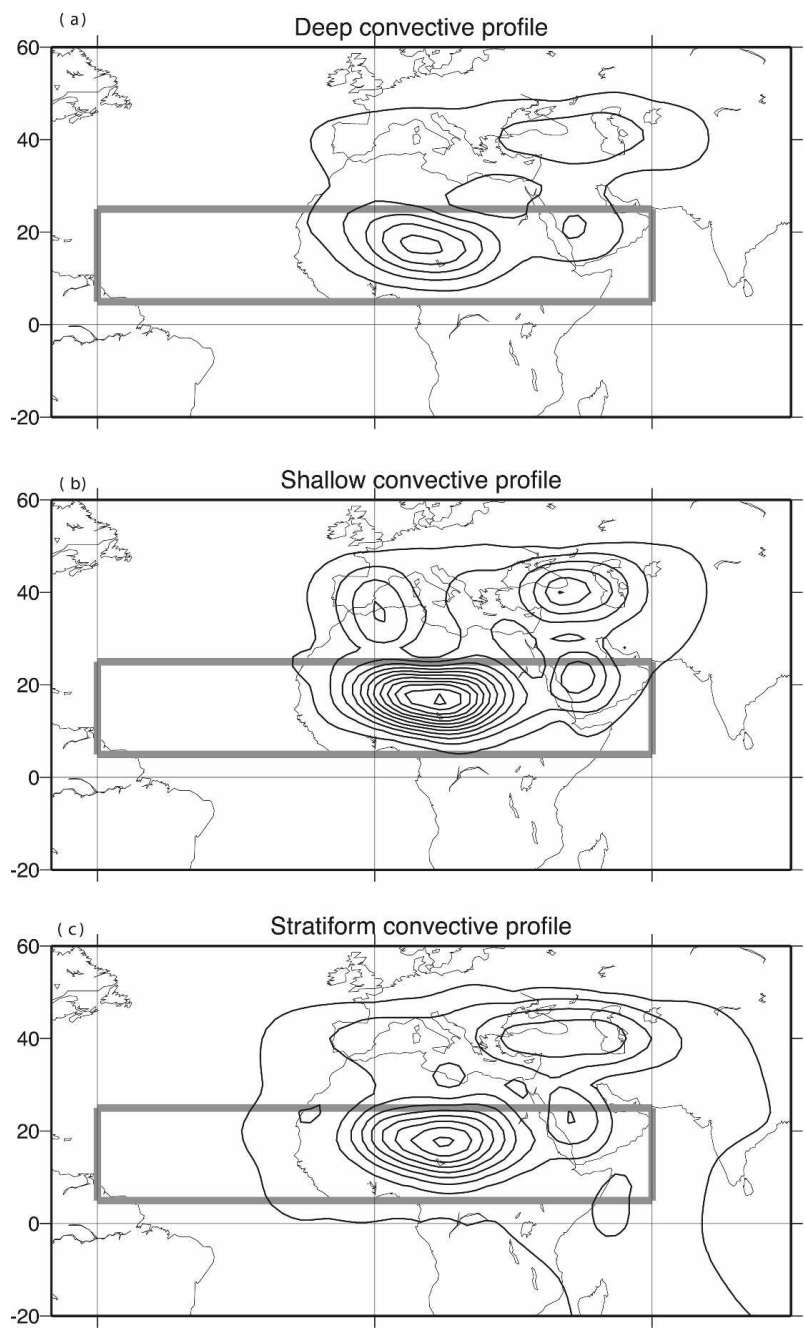

FIG. 7. Influence function for (a) deep, (b) shallow, and (c) stratiform convective anomalies, showing the most sensitive location for the initiation of AEWs that have high amplitude on day 10 in the designated rectangular area (see text for precise definition). Contours are every $10^{5} \mathrm{~m}^{2} \mathrm{~s}^{-1}$.

shifted westward into the jet and eastward away from it. The range of latitudes that leads to significant growth is also quite limited except for small localized regions located in the western and eastern Mediterranean and another over the Arabian peninsula. However, although perturbations in these latter two areas produce synoptic activity over West Africa, these do not correspond to AEW structures (not shown).

We now briefly illustrate the nature of the variability in the atmospheric response to changing the location of the heating by showing the day-7 streamfunction at $\sigma=$ 0.85 of the simulations forced by shallow heating at different locations along the axis of the AEJ. Figure 8a shows the response to heating at $20^{\circ} \mathrm{N}, 35^{\circ} \mathrm{E}$ located over the Ethiopian highlands. Mekonnen et al. (2006) 
(a) $35 \mathrm{E} 20 \mathrm{~N}$

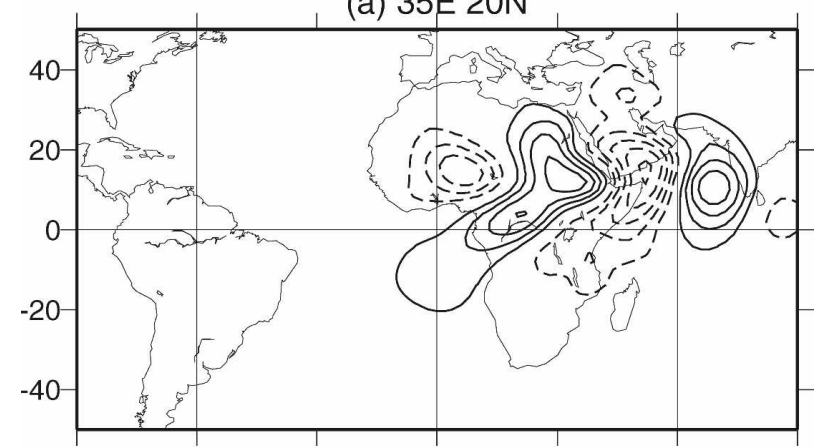

(b) $15 \mathrm{E} 20 \mathrm{~N}$

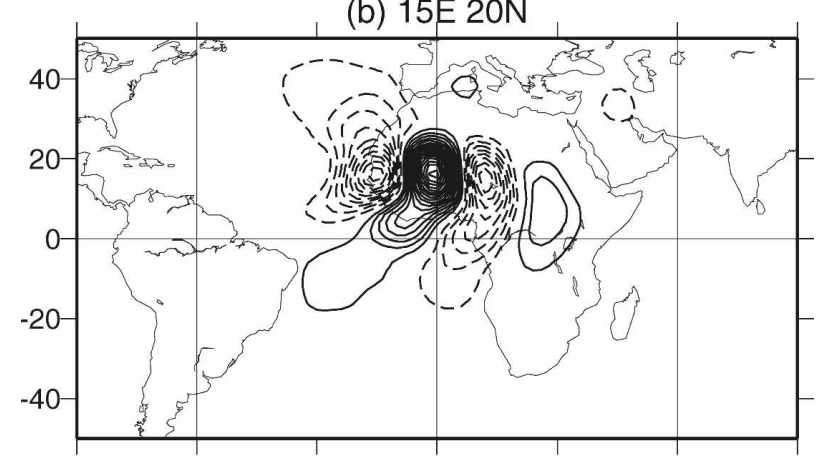

(c) $0 \mathrm{E} 15 \mathrm{~N}$

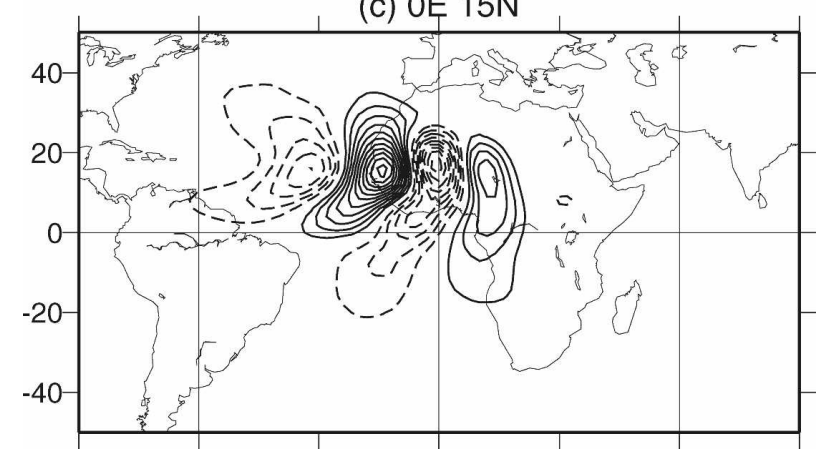

FIG. 8. Examples of day 7, $\sigma=0.85$ streamfunction response for different initial heating locations. Heating is shallow convection centered on (a) $20^{\circ} \mathrm{N}, 35^{\circ} \mathrm{E}$, (b) $20^{\circ} \mathrm{N}, 15^{\circ} \mathrm{E}$, and (c) $15^{\circ} \mathrm{N}, 0^{\circ} \mathrm{E}$. Contours are as in Fig. 5.

showed that AEWs can occasionally be triggered by convection this far to the east. For this location, the initial forced AEW trough moves westward and weakens. By day 7 it is a rather weak disturbance located just east of the Greenwich meridian. Heating in this location also appears to force a structure characterized by westward-moving easterly waves located over the Indian Ocean and eastern Africa that somewhat resembles the normal mode of the September basic state discussed in HKT06. This is suggestive of the fact that AEWs without embedded heating are likely to disperse their energy eastward and weaken in this basic state.

Figure $8 \mathrm{~b}$ shows the response to heating located at $20^{\circ} \mathrm{N}, 15^{\circ} \mathrm{E}$, the location shown above to be the most effective location for forcing. The structure at day 7 is almost identical to the simulation already shown and discussed above in Fig. 5d except for a slight phase shift (consistent with the more westward location of the initial perturbation) and a slightly larger amplitude. We should note that this location is actually just downstream of the Tibesti Mountains, and although convection can be triggered by these mountains, it is far less frequent than in the Darfur region (Hodges and Thorncroft 1997). Therefore, although it is the most efficient location for forcing AEWs, it is not likely to be the location where most AEWs are in fact forced in reality (Mekonnen et al. 2006).

For completeness we also show the response to heating located at $15^{\circ} \mathrm{N}, 0^{\circ} \mathrm{E}$, close to the AEJ maximum (Fig. 8c). By day 7 the original AEW trough has dispersed somewhat and is located in the mid-Atlantic. Over Africa, the atmospheric response is again somewhat close to the normal mode structure but is noticeably weaker than for locations closer to the jet entrance (Figs. 5d, 8b).

For locations close to the AEJ, the response is always similar: an initial AEW trough followed by the normal mode structure. As the heating is moved east or west of the jet entrance the response is weaker, particularly for the normal mode response. The response for heating shifted further north is more complicated (not shown). A more northern location tends to lead to significant baroclinic growth in the subtropical westerlies, which also tend to have some tropical reflection. So although the influence functions suggest that high-latitude forcing may affect AEW activity (using the above metric), the structures forced do not resemble AEWs as described here. Furthermore, widespread convection is relatively rare north of around $20^{\circ} \mathrm{N}$ over the Sahara, even during the monsoon.

\section{Summary and discussion}

We have shown that heating in the vicinity of the AEJ entrance, close to Darfur, forces AEWs downstream. The heating leads to an initial trough that reaches the West African coast about 5-7 days later and, following this, a structure that projects strongly onto the leading linear normal mode, characterized by a train of westward-propagating AEWs. This sequence of events is robust in this model and takes place with different heating profiles, different spatial scales, and different locations even though the amplitude of the forced AEWs is quite sensitive to these differences.

The strongest AEW response is with the shallow heating profile located close to the entrance of the 
AEJ. The deep heating profile results in a much weaker AEW response than either the shallow or stratiform heating profiles. This is the result of a much weaker initial perturbation that is established after 1 day of heating, consistent with weaker vertical gradients in heating (Hoskins et al. 1985). In reality, large-scale heating consists of varying combinations of shallow, deep, and stratiform profiles (e.g., Schumacher et al. 2004; Mapes et al. 2006). Our results suggest that the observed AEW developments in response to largescale heating will be sensitive to the relative contributions of each. It should be stressed, however, that observed AEW-associated convection, missing in these simulations, would likely intensify all AEWs regardless of how they are triggered, because latent heating has been inferred to be an important energy source for the maintenance of these waves (Thorncroft and Hoskins 1994b; Hsieh and Cook 2005, 2007). In this study we have regarded convection only as a trigger and have not addressed the subsequent question of feedback between the AEWs that are generated and further convection within the wave (Fink and Reiner 2003).

We note that for this model setup that includes lowlevel damping, this basic state is stable (HKT06). Therefore, the "natural selection" paradigm, which relies on the presence of a most unstable mode, cannot explain the modeled AEWs in our simulations. The results presented in this paper thus reinforce the growing body of work that supports the idea that AEWs are triggered by upstream heating (Carlson 1969a; Berry and Thorncroft 2005; Mekonnen et al. 2006; Kiladis et al. 2006). This view for the genesis of AEWs has consequences for how we should investigate and interpret the nature and variability of AEWs at weather and climate time scales.

Our results indicate that a significant convective outbreak in the Darfur region will favor the formation of a train of AEWs to the west over sub-Saharan Africa within a few days. Specifically, an AEW trough will reach the West African coast 5-7 days later (depending on the heating profile), which will be followed by a period of enhanced AEW activity. Thus, a number of AEWs might be expected after one significant outbreak of convection. Of course, the scale of the waves might be significantly altered by convection occurring within them, and this needs to be thoroughly investigated. Nevertheless, the notion that AEW activity can be effectively triggered by convection over the western Sudan region has relevance not only for West Africa but also for the downstream tropical Atlantic in which each AEW trough is a potential precursor for tropical cyclogenesis (e.g., Avila and Pasch 1992). Thus for daily to medium-range forecasts of AEWs, it is important to monitor, and ultimately predict, the nature of the upstream convection.

At longer seasonal to interannual time scales, in addition to considering the nature of mean AEJ (Grist et al. 2002), we should consider the nature and variability of finite-amplitude convective heating precursors. In fact, based on our results, we should expect more intense and frequent precursors to be associated with stronger AEW activity irrespective of the nature of the AEJ. Once triggered, however, we would expect a more unstable AEJ and AEW-associated convection to be associated with stronger waves (cf. Thorncroft and Hoskins 1994b; Hsieh and Cook 2005, 2007). Subseasonal variability in the AEJ may also play a role in selecting which triggering events lead to significant AEW activity. Even if the initiation mechanism is clearly separated from the question of variability in the jet, the intermittency of AEWs probably results from a collusion of these two factors.

In Part II (HKT06) of this sequence of papers, it was shown that the linear normal modes that develop on observed zonally varying basic states over tropical north Africa closely resemble the observed AEW structures shown in Part I (Kiladis et al. 2006). HKT06 also showed that in the presence of modest damping, the normal modes are in fact stable, and so it was hypothesized that finite-amplitude precursors would be necessary for the genesis of AEWs. The idealized modeling work presented here shows that this hypothesis is a reasonable one. AEWs can indeed be triggered by finite-amplitude heating in the vicinity of the AEJ, and we have shown that they are most efficiently triggered by heating that establishes lower tropospheric circulations in the AEJ entrance region. In the present study this was best achieved by the shallow and the stratiform heating profiles, both of which have significant heating gradients in the vertical in the mid-to-lower troposphere. We should note again that this mechanism for genesis of AEWs is different from that proposed by Hsieh and Cook $(2005,2007)$, who highlight the importance of upstream convection for generating an unstable PV strip (Schubert et al. 1991) and the subsequent development of AEWs on that strip. These mechanisms are not mutually exclusive and may each be important at different times, although this clearly needs further investigation.

This sequence of three papers has shed light on the nature of AEWs, including their genesis. We have established that one mechanism for the triggering of AEWs is likely due to finite-amplitude upstream heating. As the AEWs propagate along the zonally varying AEJ, they impact the environment on the synoptic scale and mesoscale, favoring new convection in differ- 
ent phases of the AEW as they propagate between the land and the Atlantic (Kiladis et al. 2006). Motivated by these studies, future work will address the important issues of AEW variability and intermittency, taking account of the nature of upstream finite-amplitude triggers, the variability of the AEJ, and the way the wave itself interacts with convective heating.

Acknowledgments. CDT was supported by NSF Grant ATM0507976. NMJH was supported by CNRS. GNK was supported by NOAA's Climate Program under Grant GC05-156. We thank Courtney Schumacher for her comments which helped to improve this paper.

\section{REFERENCES}

Albignat, J. P., and R. J. Reed, 1980: The origin of African wave disturbances during phase III of GATE. Mon. Wea. Rev., 108, $1827-1839$.

Avila, L. A., and R. J. Pasch, 1992: Atlantic tropical systems of 1991. Mon. Wea. Rev., 120, 2688-2696.

Berry, G. J., and C. D. Thorncroft, 2005: Case study of an intense African easterly wave. Mon. Wea. Rev., 133, 752-766.

Burpee, R. W., 1972: The origin and structure of easterly waves in the lower troposphere of North Africa. J. Atmos. Sci., 29, 77-90.

- 1974: Characteristics of North African easterly waves during the summers of 1968 and 1969. J. Atmos. Sci., 31, 1556-1570.

Carlson, T. N., 1969a: Synoptic histories of three African disturbances that developed into Atlantic hurricanes. Mon. Wea. Rev., 97, 256-276.

— 1969b: Some remarks on African disturbances and their progress over the tropical Atlantic. Mon. Wea. Rev., 97, 716726.

Charney, J. G., and M. E. Stern, 1962: On the instability of internal baroclinic jets in a rotating atmosphere. J. Atmos. Sci., 19, 159-172.

Dickinson, M., and J. Molinari, 2000: Climatology of sign reversals of the meridional potential vorticity gradient over Africa and Australia. Mon. Wea. Rev., 128, 3890-3900.

Eady, E. J., 1949: Long waves and cyclone waves. Tellus, 1, 33-52.

Farrell, B., 1987: Developing disturbances in shear. J. Atmos. Sci., 44, 2191-2199.

Fink, A. H., and A. Reiner, 2003: Spatio-temporal variability of the relation between African easterly waves and West African squall lines in 1998 and 1999. J. Geophys. Res., 108, 4332, doi:10.1029/2002JD002816.

Grist, J. P., S. E. Nicholson, and A. I. Barcilon, 2002: Easterly waves over Africa. Part II: Observed and modeled contrasts between wet and dry years. Mon. Wea. Rev., 130, 212-225.

Hall, N. M. J., and P. D. Sardeshmukh, 1998: Is the time-mean Northern Hemisphere flow baroclinically unstable? J. Atmos. Sci., 55, 41-56.

—_, G. N. Kiladis, and C. D. Thorncroft, 2006: Three-dimensional structure and dynamics of African easterly waves. Part II: Dynamical modes. J. Atmos. Sci., 63, 2231-2245.

Haynes, P. H., and M. E. McIntyre, 1987: On the evolution of vorticity and potential vorticity in the presence of diabatic heating and frictional or other forces. J. Atmos. Sci., 44, 828841.
Hertenstein, R. F. A., and W. H. Schubert, 1991: Potential vorticity anomalies associated with squall lines. Mon. Wea. Rev., 119, 1663-1672.

Hodges, K. I., and C. D. Thorncroft, 1997: Distribution and statistics of African mesoscale convective weather systems based on the ISCCP Meteosat imagery. Mon. Wea. Rev., 125, 2821-2837.

Hoskins, B. J., M. E. McIntyre, and A. W. Robertson, 1985: On the use and significance of isentropic potential vorticity maps. Quart. J. Roy. Meteor. Soc., 111, 877-946.

Houze, R. A., Jr., 1997: Stratiform precipitation in regions of convection: A meteorological paradox? Bull. Amer. Meteor. Soc., 78, 2179-2196.

_ 2004: Mesocale convective systems. Rev. Geophys., 42, RG4003, doi:10.1029/2004RG000150.

Hsieh, J.-S., and K. H. Cook, 2005: Generation of African easterly wave disturbances: Relationship to the African easterly jet. Mon. Wea. Rev., 133, 1311-1327.

— waves using a regional climate model. J. Atmos. Sci., 64, 421440.

Kiladis, G. N., C. D. Thorncroft, and N. M. J. Hall, 2006: Threedimensional structure and dynamics of African easterly waves. Part I: Observations. J. Atmos. Sci., 63, 2212-2230.

Mapes, B., S. Tulich, J. Lin, and P. Zuidema, 2006: The mesoscale convection life cycle: Building block or prototype for largescale tropical waves? Dyn. Atmos. Oceans, 42, 3-29.

Mekonnen, A., C. D. Thorncroft, and A. Aiyyer, 2006: Analysis of convection and its association with African easterly waves. $J$. Climate, 19, 5405-5421.

Paradis, D., J.-P. Lafore, J.-L. Redelsperger, and V. Balaji, 1995: African easterly waves and convection. Part I: Linear simulations. J. Atmos. Sci., 52, 1657-1679.

Reed, R. J., D. C. Norquist, and E. E. Recker, 1977: The structure and properties of African wave disturbances as observed during phase III of GATE. Mon. Wea. Rev., 105, 317-333.

Rennick, M. A., 1976: The generation of African waves. J. Atmos. Sci., 33, 1955-1969.

Schubert, W. H., P. E. Ciesielski, D. E. Stevens, and H.-C. Kuo, 1991: Potential vorticity modeling of the ITCZ and Hadley circulation. J. Atmos. Sci., 48, 1493-1509.

Schumacher, C., and R. A. Houze Jr., 2006: Stratiform precipitation production over sub-Saharan Africa and the tropical east Atlantic as observed by TRMM. Quart. J. Roy. Meteor. Soc., 132, 2235-2255.

- - , and I. Kraucunas, 2004: The tropical dynamical response to latent heating estimates derived from the TRMM precipitation radar. J. Atmos. Sci., 61, 1341-1358.

Simmons, A. J., 1977: A note on the instability of the African easterly jet. J. Atmos. Sci., 34, 1670-1674.

Stone, P. H., 1978: Baroclinic adjustment. J. Atmos. Sci., 35, 561571.

Thorncroft, C. D., 1995: An idealized study of African easterly waves. Part III: More realistic basic states. Quart. J. Roy. Meteor. Soc., 121, 1589-1614.

—_, and B. J. Hoskins, 1994a: An idealized study of African easterly waves. Part I: A linear view. Quart. J. Roy. Meteor. Soc., 120, 953-982.

— waves. Part II: A non-linear view. Quart. J. Roy. Meteor. Soc., 120, $983-1015$. 